\title{
Origin of the onset of Rayleigh-Bénard Convection in a concentrated suspension of microgels with a yield stress behavior
}

\author{
C. Métivier, ${ }^{\text {a) }}$ C. $\mathrm{Li}^{\mathrm{b}}{ }^{\mathrm{b}}$ and A. Magnin ${ }^{\mathrm{b}}$ )
}

(Dated: 20 October 2017)

\begin{abstract}
In this paper, we propose to explore experimentally the origin of the onset of motion in a well-known Carbopol gel, a concentrated suspension of microgels, when submitted to a vertical temperature gradient, namely the Rayleigh-Bénard Convection (RBC). We consider three possible scenario: (i) the gel behaves as an elastoviscoplastic material, (ii) the gel presents a low-stress viscosity $\mu^{+}$below the yield stress $\tau_{y}$, (iii) the gel can be considered as a two phases system, say the microgels and the solvent, i.e. a porous medium. We propose a complete rheological characterization of the Carbopol 940. Creep measurements lead to obtain an instantaneous viscosity plateau $\mu^{+} \sim t^{m}$ with $m \approx 1$. For the first time, we measure and provide permeability values $k$ in the Carbopol gels. We show that $k=O\left(10^{-14}\right) \mathrm{m}^{2}$ and $k \propto \tau_{y}^{0.2}$. Our study focuses on the reference case of the RBC with no-slip conditions at walls and new results are provided. The results lead to the conclusion that the control parameter is the (inverse) of the yield number $Y$, ratio between the yield stress and the buoyancy stress and they show that the critical value is $1 / Y_{c} \approx 80$ for no-slip conditions. One shows that both scenario (i) and (ii) lead to recover $1 / Y$ as the control parameter. By considering the Carbopol gels as porous media (scenario (iii)), one finds that the critical porous Rayleigh-Darcy number is $R a_{p}=O\left(10^{-4}\right)$
\end{abstract}

PACS numbers:

Keywords: Yield stress fluid, Carbopol gels, Rheometry, Permeability, Rayleigh-Bénard convection.

\section{INTRODUCTION}

Concentrated suspensions of microgels are widely used in large range of applications ${ }^{1}$. In particular, Carbopol gels are very popular for the last 50 years, because they are easy to prepare and use. They are considered as near ideal yield stress fluids in the rheology and in the fluid mechanics areas since they present a clear yield stress $\tau_{y}$, below which they behave mainly as an elastic solid and above $\tau_{y}$, they present a shear thinning behaviour, even at low weight concentrations $C$ of Carbopol. The yield stress is due to the microstructure of the Carbopol gels which is a percolated, amorphous assembly of soft jammed particles, named as microgels ${ }^{2-4}$. These microgels consist in highly cross-linked regions where polymers are bound together leading to dense domains. Carbopol gels have received increase attention this last decade ${ }^{4-17}$ in order to understand their structure and their fluidization mechanisms. This understansding is of utmost importance since the solid-liquid transition is responsible for the onset of motion in lot of viscoplastic fluids. A situation where this transition is involved, is the RayleighBénard convection (RBC). This configuration is of great interest since numerous industrial processes as well as natural settings involve natural convection, e.g. the mantle convection in Earth. The onset of motion in the RBC

\footnotetext{
a)Christel.Metivier@univ-lorraine.fr; Laboratoire d'Energétique et de Mécanique Théorique et Appliquée, UMR 7563 (Université de Lorraine, CNRS), France

b) Laboratoire Rhéologie et Procédés - Univ. Grenoble Alpes, CNRS, Grenoble INP*, LRP, F-38000 Grenoble, France.

*Institute of Engineering Univ. Grenoble Alpes
}

is driven by the buoyancy involved by a temperature difference $\Delta T$ between the lower (hot) wall and the upper (cold) wall. In the case of Newtonian fluids ${ }^{18-20}$, convection occurs when the buoyancy effects overcome the viscous and thermal diffusive effects, leading to the Rayleigh number $R a$ as the control parameter:

$$
R a=\frac{\rho g \beta \Delta T d^{3}}{\mu \kappa}
$$

where $\rho$ is the density, $g$ the gravitational acceleration, $\beta$ the thermal volume expansion, $d$ the fluid layer, $\mu$ the viscosity and $\kappa$ the thermal diffusivity. Few studies have been devoted to the RBC in yield stress fluids. Theoretical studies ${ }^{21-23}$ have considered an inelastic yield stress fluid (Bingham model) and show that for a finite yield stress the motionless solution is linearly stable at all $R a$ values and is conditionally stable otherwise. These conclusions mean that the RBC cannot occur in viscoplastic fluids regarding small amplitude perturbations. This is in contradiction with recent experimental results ${ }^{24,25}$ which have observed the onset of convection in a yield stress fluid without adding any perturbation. The first experimental study ${ }^{24}$ show that the control parameter is the (inverse) of the yield number $Y$, ratio between the buoyancy stress and the yield stress:

$$
Y^{-1}=\frac{\rho g \beta \Delta T d}{\tau_{y}} .
$$

Darbouli et $a .^{24}$ show that criticality depends on the boundary conditions. A recent review on slippage ${ }^{26}$, in the particular case of microgel suspensions, highlights the importance to take into account this phenomenon. Based on an extensive work, Darbouli et al. show that for 
slip conditions ' $\mathrm{S}$ ' in the Rayleigh-Bénard cavity (use of glass, copper alloy and PMMA walls), criticality is such as $1 / Y_{c}^{S} \approx 40$. Darbouli et al. show that by defining the control parameter $Y$ with the yield stress at the interface $\tau_{f}$ instead of the bulk yield stress $\tau_{y}$, the results converge to $1 / Y_{c} \approx 80$. The case of no-slip conditions 'NS' was only considered for one experiment ${ }^{24}$, leading to $1 / Y_{c}^{N S} \approx 80$.

Further experiments in the case of slip conditions have been proposed by Kebiche et al. ${ }^{25}$ which confirm that $1 / Y$ is the adequate control parameter. However, the criticality is significantly smaller $\left(1 / Y_{c}^{S} \approx 5\right)$ which is certainly due to their boundary conditions: slippage at walls and weak conductivity at the horizontal boundaries. These conditions are all known to decrease significantly critical conditions in Newtonian fluids ${ }^{34-36}$. However it is difficult to compare quantitatively these two studies since Kebiche $e t ~ a l .{ }^{25}$ do not evaluate clearly neither the level of the gels slippage at walls nor the walls conductivity in their experiments.

In the field of natural convection in Carbopol gels, one can also highlight the experimental work of Davaille et $a .^{27}$. This work investigates the onset of thermal plumes when a viscoplastic fluid layer is submitted to a localized heating. The authors show that convection occurs around the heater above a critical value $1 / Y_{c 1}=$ $120( \pm 15)$ and plumes develop above $1 / Y_{c 2}=260( \pm 20)$. The value of $1 / Y_{c 1}$ is numerically confirmed by Karimfazli et al. ${ }^{28}$.

In spite of these recent works ${ }^{24,25,27}$, the origin of onset of motion in Carbopol gels when heated from below is still not well understood. Darbouli et al. ${ }^{24}$ proposed three possible scenario which could be at the origin of the onset of the RBC in Carbopol gels. The first scenario (i) consists in considering the Carbopol gels as elastoviscoplastic materials, especially below and close to the yield stress. This scenario received a first consideration in Darbouli et $a .^{24}$ in the RBC case with slip conditions at walls and Davaille et al. ${ }^{27}$, in the case of thermal plumes. The second scenario (ii) is a viscous scenario, it consists in considering a finite viscosity at low values of shear rates. This scenario has been numerically investigated by Massmeyer et al. ${ }^{29}$ in the case of thermal plumes and by Li et al. ${ }^{30}$ in the RBC case. These studies consider regularized models of a Herschel-Bulkley fluid. In the first study ${ }^{29}$, the authors consider the regularization proposed by de Souza Mendes \& Dutra ${ }^{31}$ and in the second study ${ }^{30}$, the Papanastasiou regularization is considered. The use of regularized models can provide information however they present some problems regarding the regularized parameter ${ }^{32,33}$, furthermore they are not realistic models. This is all the more true when regarding recent studies ${ }^{10,14,15}$ which have provided rheological measurements in Carbopol gels below the yield stress. In these studies, an instantaneous viscosity plateau $\mu^{+}$has been measured below the yield stress. The value of $\mu^{+}$ is defined as the ratio between the imposed stress $\tau$ and the measured shear rate $\dot{\gamma}$. It has been shown that $\mu^{+}$ depends on the delay time: $\mu^{+} \approx t^{m}$. It means that below the yield stress, the Carbopol gels do not lead to a constant (Newtonian) viscosity but to a constant value of viscosity at fixed time. The third proposed scenario (iii) would consist in considering the microstructure of the gels, i.e. a structure composed by percolated elastic microgels and pools of solvent all around. In this sense, the motion could first occur in these pools of a Newtonian solvent (water) inducing then a sufficient level of stress in the bulk up to yield the whole material. In this scenario, one would consider the Carbopol gels as a particular class of porous media.

While the scenario (i) has to be considered in the case of no-slip conditions, the scenario (ii) and (iii) have never been investigated.

This present paper aims to investigate the origin of the onset of the RBC in Carbopol gels. For this, we propose to study the different scenario (i)-(iii) outlined above and especially the scenario (ii)-(iii). The section 'Materials and Rheometry' is dedicated to present the structure of our working system as well as its rheometry. In order to explore the scenario (iii) which considers the gels as porous media, one section is dedicated to present permeability measurements in our Carbopol gels. To our best knowledge, this is the first time that permeability of Carbopol gels is provided. Because the reference case of the RBC in Carbopol gels with no-slip conditions has been understudied (only one experiment has been performed), our study focuses on this case and new results are provided in the section 'Rayleigh-Bénard convection'. Finally all scenario are discussed in the last section which also includes a conclusion.

\section{MATERIALS AND RHEOMETRY}

Carbopol gels are considered as simple yield stress fluids ${ }^{8,9}$ by contrast with that ones which present thixotropy. Carbopol is a polymer made of crosslinked polyacrylic acid. When mixed with water, a turbid dispersion is obtained with an acid $\mathrm{pH}$. The yield stress appears when the dispersion is neutralized by adding sodium hydroxide around $\mathrm{pH}=7$. During this process, the carboxyl group is ionized leading to repulsive forces. Molecules swell in the whole space due to an outward osmotic pressure in the presence of ions ${ }^{37}$. The swollen microgels size is about $2-20 \mu \mathrm{m}^{4,7,13}$, depending on the $\mathrm{pH}$, the ionic strength, the degree of ionisation and the concentration.

In this present study, we use the Carbopol 940 from BF Goodrich in the range of concentrations $0.035 \%<$ $C<0.12 \%$. In this concentration domain, the material consists in a weak percolated structure containing pools of solvent and in fully swollen microgels. Because the weight concentration of the microgel is sensitive to many parameters, our gels will be refered to their rheological properties, in particular their respective yield stress value $\tau_{y}$ ranging from $0.02 \mathrm{~Pa}$ to $6 \mathrm{~Pa}$ as indicated below. The 


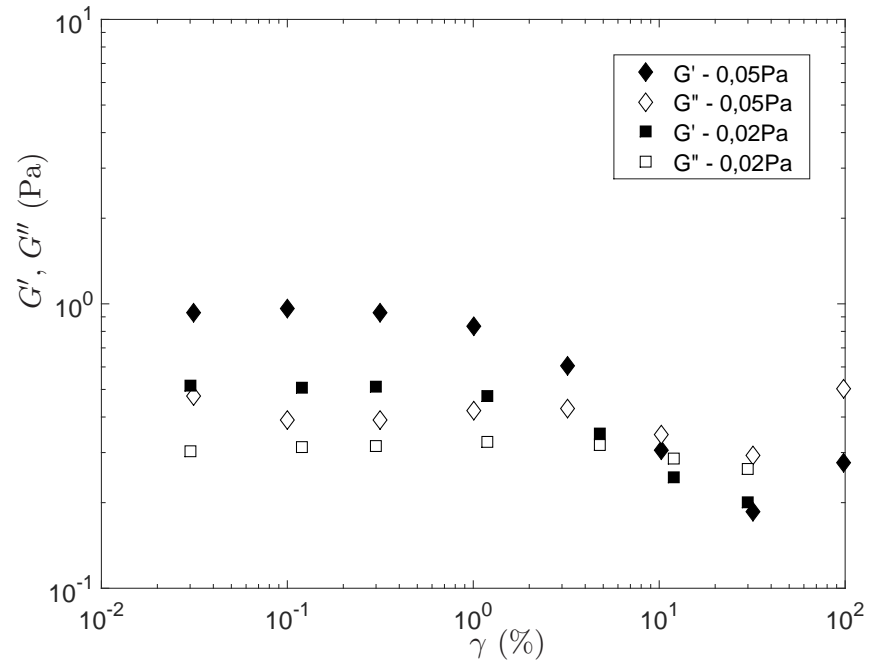

FIG. 1. Elastic modulus (full symbols) and loss modulus (empty symbols) as a function of the strain for two Carbopol gels $\left(\diamond: \tau_{y}=0.05 \mathrm{~Pa}\right.$ and $\left.\square: \tau_{y}=0.02 \mathrm{~Pa}\right)$ at $\omega=10 \mathrm{rad} / \mathrm{s}$.

Carbopol gels present an elasto-viscoplastic behaviour. Below the yield stress, a linear viscoelastic regime is observed under small amplitude oscillatory shear (SAOS) measurements, highlighting a solid elastic response regarding small deformation. The Figure 1 displays the elastic modulus $G^{\prime}$ and the loss (viscous) modulus $G^{\prime \prime}$ as a function of the strain $\gamma$ for two different Carbopol gels. The linear domain is defined for $\gamma<1 \%$, where $G^{\prime}$ and $G^{\prime \prime}$ are constant. In this regime, the Carbopol gels present a dominant elastic behaviour since $G^{\prime}>G^{\prime \prime}$. For larger strain values, the moduli decrease and they cross at a critical strain value $\gamma_{c}$. For our Carbopol gels, we find $\gamma_{c} \approx 10 \%$. The stress measured at this particular value of strain, i.e. $\tau_{c}=G^{\prime} \gamma_{c}$, is a good estimation of the yield stress $\tau_{y}$. One finds $\tau_{c}=0.047 \mathrm{~Pa}$ (resp. $\left.\tau_{c}=0.022 \mathrm{~Pa}\right)$ for the gel characterized by $\tau_{y}=0.05 \mathrm{~Pa}$ (resp. $\tau_{y}=0.02 \mathrm{~Pa}$ ) with a steady-state rheometry.

In SAOS rheometry, $G^{\prime}$ and $G^{\prime \prime}$ are measured by varying the oscillation pulsation $\omega$ (or frequency) in the linear regime. In this respect, we fix the strain to $0.5 \%$ which implies a maximal stress value $0.01 \mathrm{~Pa}\left(<\tau_{y}\right)$. As given in Fig. 2, the Carbopol, characterized by $\tau_{y}=0.05 \mathrm{~Pa}$, displays a dominant elastic modulus for all values of $\omega$ and $\gamma=0.5 \%$. The elastic modulus $G^{\prime}$ is found one order larger than the viscous modulus $G$ ". Similar tendencies are observed for all the Carbopol gels used.

Above the yield stress, the Carbopol flow curves are well fitted by the Herschel-Bulkley (HB) model given by

$$
\tau=\tau_{y}+K \dot{\gamma}^{n}
$$

where $\dot{\gamma}$ is the shear rate, $K$ is the consistency and $n=0.3-0.6$ the shear thinning coefficient. Flow curves of the Carbopol gels used in our study are obtained by performing a steady-state rheometry. Results are shown in Fig. 3. Results are nicely fitted by the HerschelBulkley model given by Eq. (3) and represented by

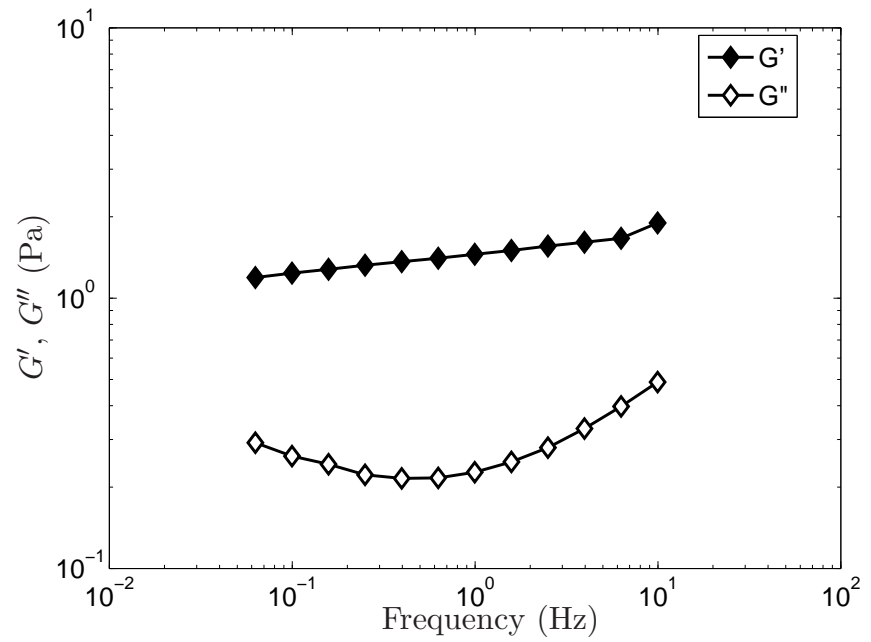

FIG. 2. Elastic and loss moduli for a Carbopol gel $\left(\tau_{y}=0.05\right.$ Pa) at $\gamma=0.5 \%$.

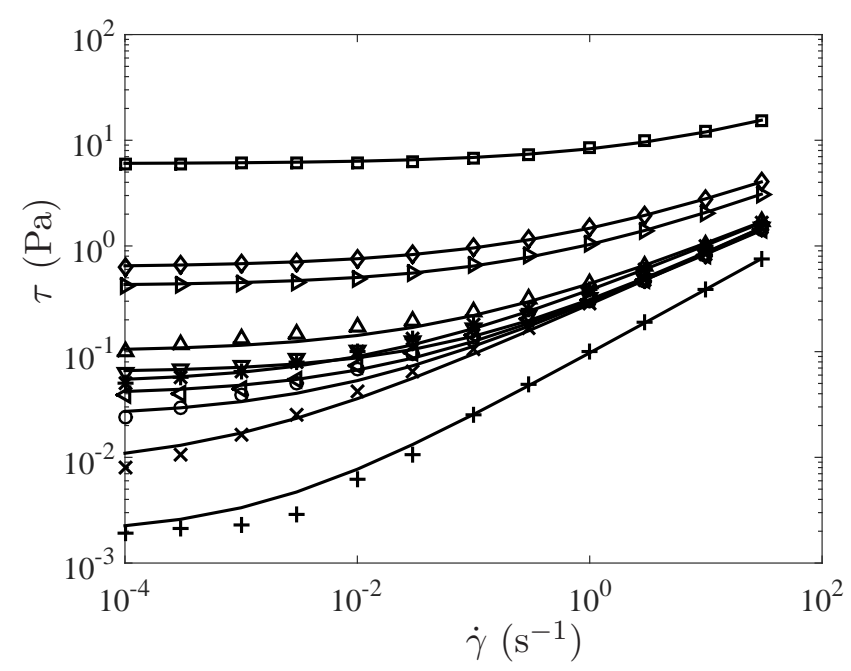

FIG. 3. Steady-state flow curves of different Carbopol gels $\tau_{y}=0.002 \mathrm{~Pa}, 0.008 \mathrm{~Pa}, 0.02 \mathrm{~Pa}, 0.039 \mathrm{~Pa}, 0.05 \mathrm{~Pa}, 0.063$ $\mathrm{Pa}, 0.1 \mathrm{~Pa}, 0.42 \mathrm{~Pa}, 0.63 \mathrm{~Pa}, 6 \mathrm{~Pa}$ (from + to $\square$ symbols) straight lines: Herschel-Bulkley model.

straight lines. At low Carbopol concentrations (low yield stress values), one can observe some discrepancies between the Herschel-Bulkley model and the experimental data. These discrepancies are due to the increase in the measurement errors when the yield stress values are weak. In the following, we fix the yield stress value of our Carbopol gels to the value measured at $\dot{\gamma}=10^{-4}$ $\mathrm{s}^{-1}$, which is also a value fixed in the Herschel-Bulkley fitting procedure.

\section{Fluidization mechanisms}

This last decade, several studies ${ }^{5,6,10,12}$ have explored the fluidization mechanisms in Carbopol gels under 
imposed shear stress. This situation is of great interest to our study since it is involved in the natural convection experiments where buoyancy stresses are increased from a motionless state (conductive regime) to the onset of motion (convection regime). When the imposed shear stress is larger than the residual stresses $\tau_{r}$, these surveys observe three regimes before reaching the steady flow regime modeled by the $\mathrm{HB}$ law.

(1) A first regime corresponds to an elastic response of the material over short times whatever the applied stress.

(2) Then, the microgels experience a creep deformation below the yield stress. It is shown that the deformation rate present a slow decay characterized by a power law $\dot{\gamma} \propto t^{-p}$, similar to an Andrade's law as observed in solid materials ${ }^{39,40}$. Values of $p$ vary depending on the literature, i.e. $p=2 / 3$ in the case of Carbopol ETD 2050 gels $^{12,15}$ while $p=1$ in the case of hair gels, Carbopol 940 gels and composite materials ${ }^{5,6,40}$. The Andrade's creep finds its origin in the dynamics of dislocations of soft metals microstructure. After several studies $^{41,42}$, heterogeneous materials as well as jammed systems (e.g. gels, foams, granular media) exhibit similar mechanisms as the metals such as the primary creep regime. Furthermore, Moller et $a l^{10}$ have also measured a creep below the yield stress characterized by a low-stress viscosity plateau $\mu^{+}$which depends on the delay time following a power law $\mu^{+} \propto t^{m}$, with $m=0.6$ in the case of Carbopol Ultrez U10 gels. This viscosity corresponds to an instantaneous value since no steady-state shear is observed in this regime. By performing large amplitude oscillatory shear stress (LAOStress), Dimitriou et $a l^{14}$ find a similar tendency with $m=0.9$ in the Carbopol 901. The authors show that this apparent viscosity $\mu^{+}$can be predicted by the kinetic hardening since the Carbopol is not a perfect elastic solid below the yield stress. Kinetic hardening accounts for dynamical movement of the yield surface (in stress space), involving that the yield stress of the material evolves dynamically with time as the material is deformed.

Similarly, we have performed creep measurements below yielding, i.e. a shear stress is imposed and the shear rate is recorded (Fig. 4). Different time measurements (1200 s, 2000 s, 3600 s, $10800 \mathrm{~s}$ ) have been performed in order to investigate the time dependency of results below yielding. These creep times are the same order of typical times involved in our Rayleigh-Bénard experiments. A result of particular interest is the measurement of an apparent viscosity $\mu^{+}=\tau / \dot{\gamma}$ below the yield stress $\tau_{y}$ (Figs. 4 and 5). As obtained in the literature, the apparent viscosity displays a constant value which varies with time as $\mu^{+} \sim t^{m}$ (Fig. 5 ), where $m \approx 1$ within our measurements resolution. This value is close to the ones obtained by Dimitriou et al. ${ }^{14}(m=0.9)$, by Coussot et $a l . .^{5}$ and Caton \& Baravian $^{6}(m=1)$. When the applied shear stress $\tau_{a}$ is larger than $\tau_{y}$, the steady-state regime is reached and follows the HB law as shown in Figs. 4

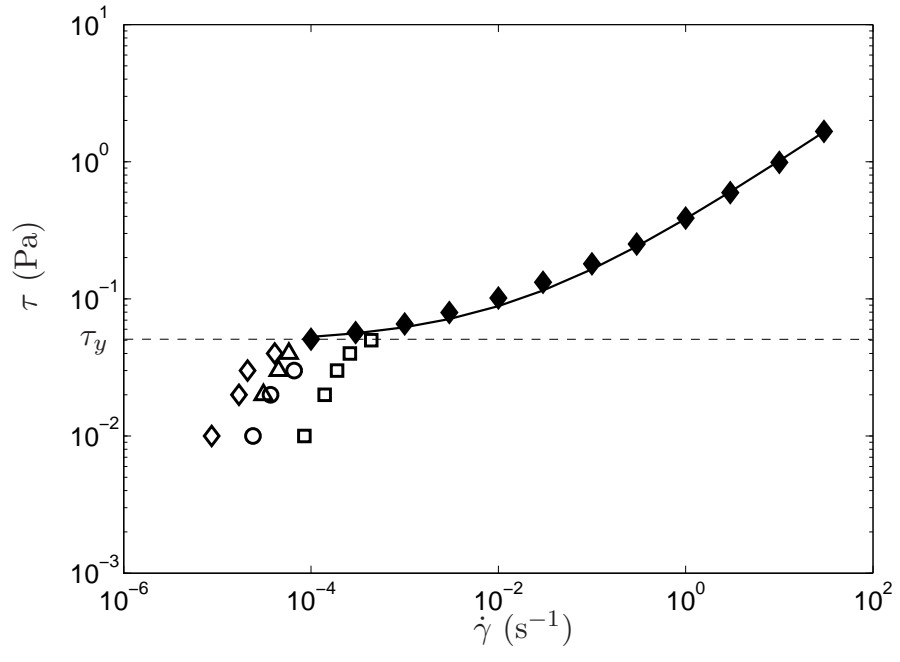

FIG. 4. Creep measurements for different time tests and one Carbopol gel $\left(\tau_{y}=0.05 \mathrm{~Pa}\right)-\diamond: 10800 \mathrm{~s}, \triangle: 3600 \mathrm{~s}, \bigcirc$ : $2000 \mathrm{~s}, \square: 1200 \mathrm{~s}-\downarrow$ : steady-state measurements for $\tau_{a}>\tau_{y}$, continuous line: Herschel-Bulkley model.

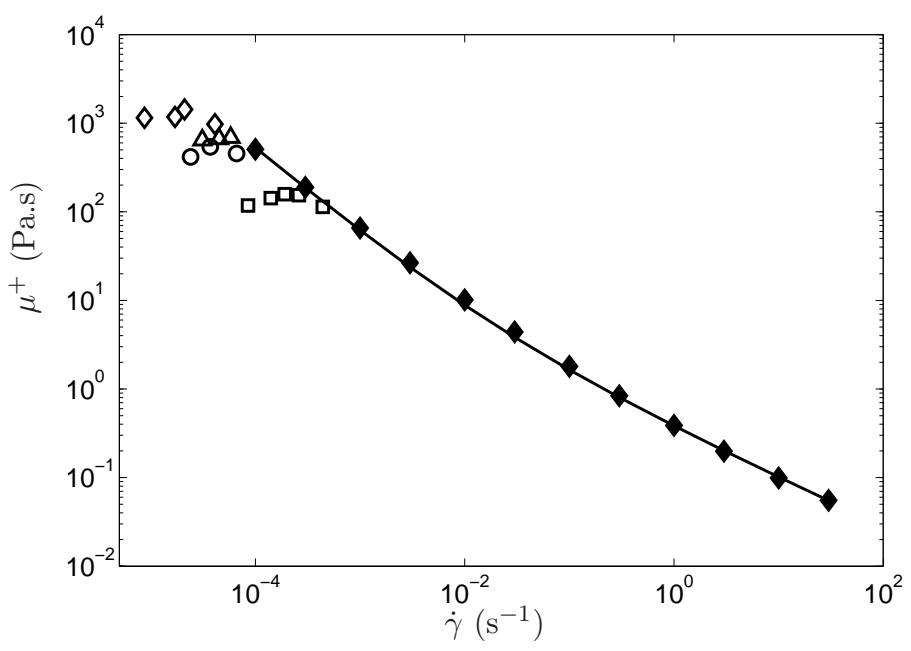

FIG. 5. Viscosity $\mu^{+}$as a function of shear rate obtained by creep measurements for different time tests and one Carbopol gel $\left(\tau_{y}=0.05 \mathrm{~Pa}\right)-\diamond: 10800 \mathrm{~s}, \triangle: 3600 \mathrm{~s}, \bigcirc: 2000 \mathrm{~s}, \square$ : $1200 \mathrm{~s}-\$$ : steady-state measurements for $\tau_{a}>\tau_{y}$, continuous line: Herschel-Bulkley model.

and 5 .

One can note that when the applied stress is such that $\tau<\tau_{r}\left(<\tau_{y}\right)$, a recent study ${ }^{15}$ shows that residual stresses in the Carbopol gel can play a significant role and can lead to 'anomalous' creep response, meaning that relaxing residual stresses tend to deform the gel in the opposite direction to that of preshear. In there experiments, Lidon et al. ${ }^{15}$ estimate the level of residual stresses around $20 \%$ of the yield stress. Residual stresses have already been observed by $\mathrm{Piau}^{4}$ and Coussot ${ }^{5}$, who show that Carbopol gels can experience a significant 


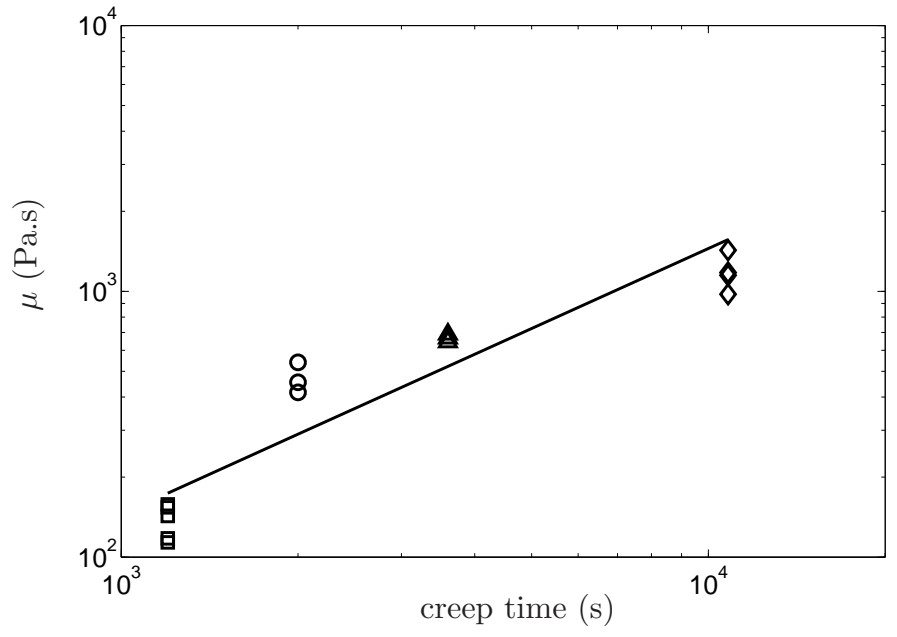

FIG. 6. Viscosity as a function of the creep time obtained by creep measurements for one Carbopol gel $\left(\tau_{y}=0.05 \mathrm{~Pa}\right)-\diamond$ : $10800 \mathrm{~s}, \triangle: 3600 \mathrm{~s}, \bigcirc: 2000 \mathrm{~s}, \square: 1200 \mathrm{~s}$, continuous line: slope $1(m=1)$.

level of stress relaxation. This phenomenon can have significant consequences as highlighted in the context of buble rise ${ }^{38}$ for instance.

(3) At the end of the Andrade's creep regime, a minimal value of shear rate is observed ${ }^{5,6,12}$, then for larger time, $\dot{\gamma}$ increases in a more or less abrupt way until the steadystate flow is reached. This regime corresponds to the total fluidization of the Carbopol gel. It is characterized by a total wall slip even with rough boundary conditions and by a transient shear banding which nucleates at the rotor and extends across the gap ${ }^{11,12}$. Full fluidization of the material is reached at the time $t_{f}$ after which the velocity profiles across the gap remain homogeneous and steady. Divoux et al. ${ }^{11,12}$ show that $t_{f} \sim 272 / \dot{\gamma}^{2.3}$ in their working system and also that $t_{f}=D\left(\tau-\tau_{y}\right)^{\beta_{1}}$ where $D$ and $\beta_{1}$ depend on the Carbopol concentration.

\section{GELS PERMEABILITY MEASUREMENTS}

\section{A. Experimental setup}

The Carbopol gels sample of height $e$ is contained in a cylindrical cell, closed at the upper and lower parts by two membranes made of a mixed cellulose ester (MFMillipore, ref. GSWP04700). The size of the membrane pores is $0.22 \mu \mathrm{m}$, i.e. at least one order smaller than the microgels size (around 2-20 $\mu \mathrm{m}$ ). In addition, two millipore filters support the membranes and the porous medium. Similarly to Ambari et al. ${ }^{43}$, who measured the permeability in deformable porous media such as gels, we use two vertical and parallel capillary tubes in order to generate the flow through the gel sample via an initial difference in height $\Delta H_{0}$ leading to a pressure drop $\Delta P$.
Here, the tubes are connected to the upper and lower parts of the cell. The whole system is full of solvent (water). The level of the capillary tube is measured with an accuracy of $100 \mu \mathrm{m}$.

At steady state, the permeability $k$ is given by the Darcy's law:

$$
k=\frac{Q \mu e}{S \Delta P}
$$

where Q corresponds to the water flow rate through the porous medium (here Carbopol microgels), $\mu$ is the viscosity of the solvent (water) and $S$ is the cross-sectional area to flow. The flow rate $Q$ is evaluated via the height variation $h(t)$ of the meniscus in one capillary tube: $Q=\frac{h(\delta t) \Sigma}{\delta t}$, with $\Sigma$ the diameter of the capillary and $\delta t$ the measurement time. The pressure drop $\Delta P$ is evaluated as follows:

$$
\Delta P=\rho g\left(\Delta H_{0}-2 h(t)\right) .
$$

One notices that measurements done without any gel in the cell show that the hydraulic resistance ${ }^{44} R_{h}$ of the setup is two orders of magnitude smaller than the Carbopol gels hydraulic resistance. In this way, the intrinsic resistance of the setup has been neglected in Eq. (5).

\section{B. Permeability measurements}

Two different fluids have been considered to flow through the Carbopol gels. The first solvent is distilled water (circles and squares in Fig. 7), the second one is the liquid phase (triangles in Fig. 7) extracted directly from a Carbopol gel. This latter fluid has a similar physicochemical composition to that of the Carbopol gels. The fluid phase has been extracted from a Carbopol gel by using a $0.22 \mu \mathrm{m}$ filter cell under pressure. No significant difference between the two solvents was observed as shown in Fig. 7.

Mougin et $a l .{ }^{38}$ have shown that the trajectory of quasi static bubles in Carbopol gels present a preferential path due to residual internal stresses in the gels. In the permeability measurements, the existence of such preferential paths for the solvent flow could modify significantly the $k$ values. In this sense, we have verified that the flow is homogeneous in the whole volume of the setup by introducing a colored solvent in the gel. One observed that the colored water diffuses homogeneously in the whole sample. The independence of $k$ values with $\Delta H_{0}$ has also been verified by testing different values of initial height $(2$ $\mathrm{cm}$ and $12 \mathrm{~cm})$. For larger $\Delta H_{0}$ values such as $\Delta H_{0}=60$ $\mathrm{cm}$, microgels deform and one observes a large compacting of microgels. The compacting is such that one can observe an interface where the microgels concentration is very large. It traduces that our microgels suspension consists in a deformable medium when submitted to pressure. The initial pressure corresponding to $\Delta H_{0}=60$ is close to $6000 \mathrm{~Pa}$. 


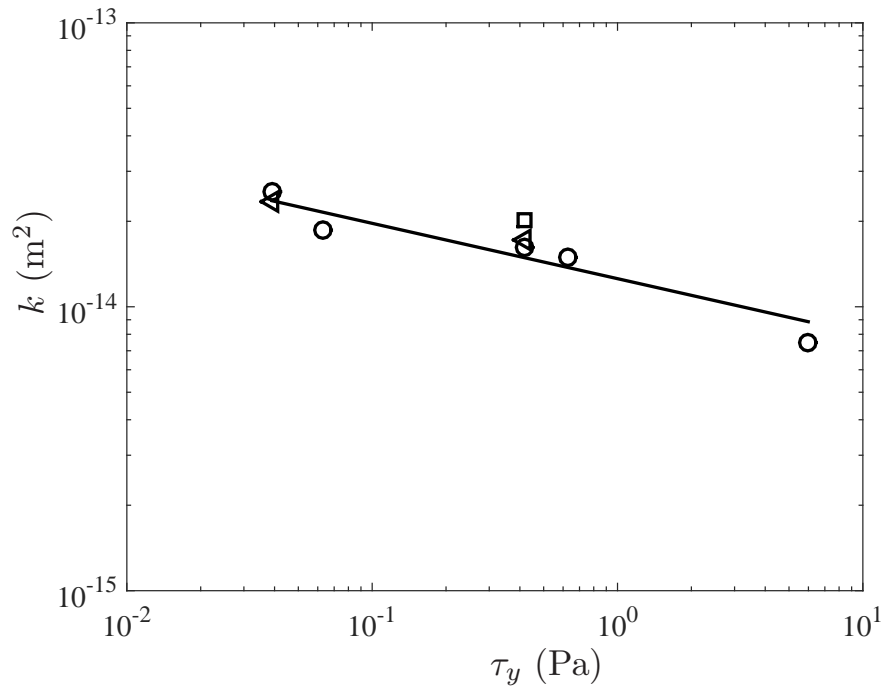

FIG. 7. Permeability as a function of the yield stress - $\bigcirc$ : $\Delta H_{0}=2 \mathrm{~cm}$, distilled water ; $\square: \Delta H_{0}=12 \mathrm{~cm}$, distilled water ; $\triangleleft: \Delta H_{0}=2 \mathrm{~cm}$, Carbopol liquid phase as the solvent, continuous line: slop -0.2 .

The gels permeability as a function of the yield stress is displayed in Fig. 7. As expected, one observes that the permeability decreases with increasing values of $\tau_{y}$ which is correlated with the increase in the microgels concentration. In the range of tested gel concentrations, one obtains $k \propto \tau_{y}^{-0.2}$. One can notice the relatively weak values of permeability since $k=O\left(10^{-14}\right) \mathrm{m}^{2}$. These values agree with the permeability values obtained in other gels such as polyacrylamide gels ${ }^{45-47}$ and agarose gels ${ }^{48}$. These results confirm that the cross-linked regions (microgels) represent a non-draining or weak-draining core and that microgels are surrounded by a rather dilute freely draining zone as also observed in polyacrylamide gels $^{45-47}$.

In their article, Ketz et $a ._{.}^{2}$ proposed a way to evaluate the permeability of the Carbopol gels by considering dimensional and structural arguments, leading to the following relationship:

$$
k=\frac{\mu R^{2}}{G t_{c}}
$$

where, $\mu$ is the solvent viscosity, $R$ is the microgel radius, $G$ is the modulus of the microgels network and $t_{c}$ is the characteristic time for the volume equilibration after the gel network have been deformed which lead to changes in the microgels volume. Below the yield stress, one can evaluate from our measurements (see Fig. 1) $G \approx G^{\prime}=$ $O(1) \mathrm{Pa}$ and $t_{c}=O(10) \mathrm{s}$. The solvent viscosity is that of water, i.e. $\mu=10^{-3}$ Pa.s and one can estimate a mean radius of the microgels ${ }^{4,7,13} R=O\left(10^{-5}\right) \mathrm{m}$. By considering these values, one can obtain an estimation of the permeability $k \approx 10^{-14} \mathrm{~m}^{2}$ which agrees with our measurements.

\section{RAYLEIGH-BÉNARD CONVECTION}

The Rayleigh-Bénard cavity is a circular cell of $D=$ $179 \mathrm{~mm}$ as the diameter and three different height values $d=10,17$ and $30 \mathrm{~mm}$ corresponding respectively to aspect ratio values $\Gamma(=D / d)$ of 18,10 and 6 . The total heat flux $Q_{t}$ of the bottom wall is controlled by means of an electrical heater plate, the temperature upper wall is controlled via a (cold) water flow. The Polymethyl Methacrylate (PMMA) side walls of $30 \mathrm{~mm}$ thickness involve adiabatic conditions. Further technical details of the setup can be obtained in Darbouli et al. ${ }^{24}$. No-slip conditions at walls have been obtained by treating the cavity surfaces with a solution of polyethylenemine (PEI) in water ${ }^{53}$. This treatment is very usefull since it allows to obtain no-slip conditions on hydrophobic, smooth and transparent walls such as glass (borosilicate) or PMMA walls, without modifying their transparency ${ }^{53}$. Four Carbopol gels $\left(\tau_{y}=0.002 \mathrm{~Pa}, 0.008 \mathrm{~Pa}, 0.02 \mathrm{~Pa}\right.$ and $\left.0.05 \mathrm{~Pa}\right)$ have been tested in the Rayleigh-Bénard setup. Temperature measurements are done at any time by thermocouples. The onset of convection is determined by using the Schmidt-Milverton principle ${ }^{54}$ which considers that in the conductive regime, $Q_{t}$ is proportional to the horizontal walls temperature difference $\Delta T=T_{2}-T_{1}$. This curve variations evolve significantly with the onset of convection. The convection tends to homogenize the temperature in the cavity, meaning that the variations of $\Delta T$ vs. $Q_{t}$ lead to a decrease in the curve slope as one can observe in Fig. 8 for different cavity height $d$. One can notice that each value displayed in Fig. 8 has been obtained at steady state. At each change in $Q_{t}$ values, we wait several hours up to 10 hours in order to reach the steady state. In the conductive regime, one waits around 2 hours to ensure that the linear profile of temperature is reached in the fluid layer. At the onset of convection and in the convective regime one waits 6 to 10 hours.

In order to highlight the governing parameter, values of $\beta \Delta T_{c} / \tau_{y}$ are displayed as a function of $d$ in Fig. 9 . Similarly to Darbouli et $a .^{24}$, one obtains $\beta \Delta T / \tau_{y} \propto$ $d^{-1}$, meaning that the governing dimensionless number is the (inverse) of the yield number as defined in Eq. (2). For all this set of experiments, one obtains:

$$
Y_{c}^{-1}=80 \pm 15
$$

The physical origin of this number is discussed below considering the scenario (i)-(iii).

\section{v. DISCUSSION AND CONCLUSION}

i) The first proposed scenario has been well developed in Darbouli et $a .^{24}$ in the slip case by considering an elasto-plastic material, i.e. a solid with an elastic response then a plastic response when the stress (or strain) increases till $\tau_{y}$. In the elastic regime, the internal stress writes $\sigma=G^{\prime} \gamma$. As shown in the SAOS 


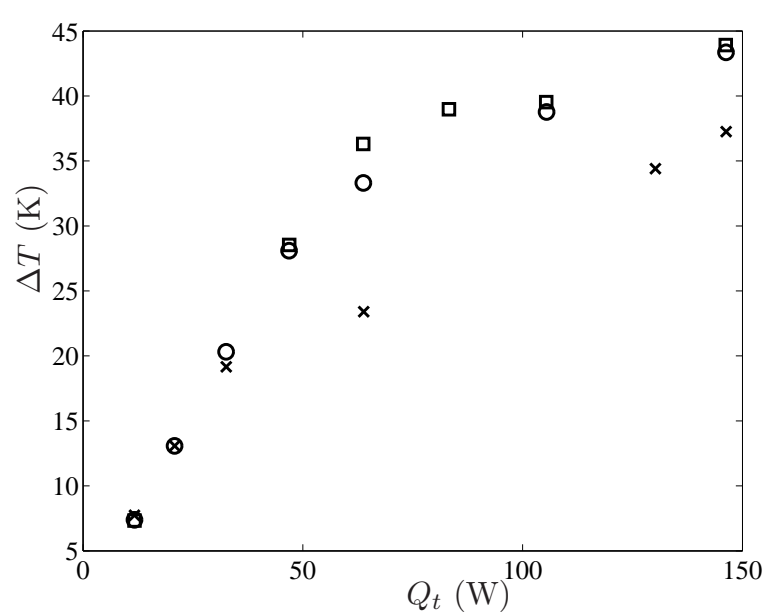

(a) $d=10 \mathrm{~mm}$

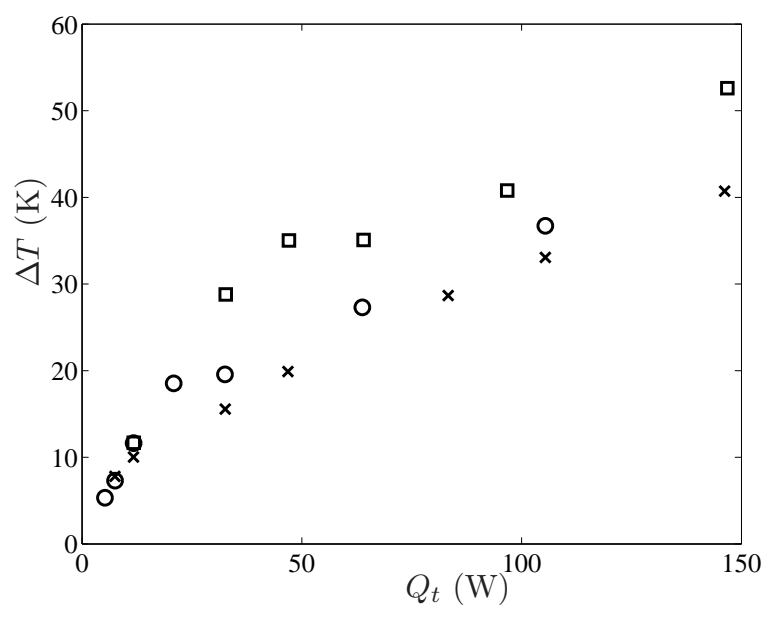

(b) $d=17 \mathrm{~mm}$

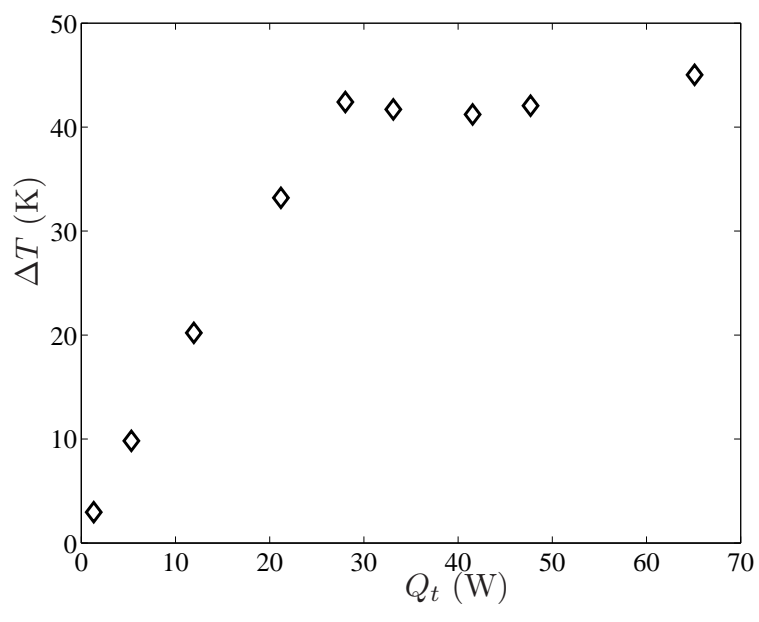

(c) $d=30 \mathrm{~mm}$

FIG. 8. Variations of $\Delta T$ as a function of $Q_{t}$ for different Carbopol gels $\left(\diamond: \tau_{y}=0.05 \mathrm{~Pa}, \square: \tau_{y}=0.02 \mathrm{~Pa}, \bigcirc: \tau_{y}=\right.$ $0.008 \mathrm{~Pa}, \times$ : $\tau_{y}=0.002 \mathrm{~Pa}$ ) and for (a) $d=10 \mathrm{~mm},(\mathrm{~b})$ $d=17 \mathrm{~mm}$ and (c) $d=30 \mathrm{~mm}$.

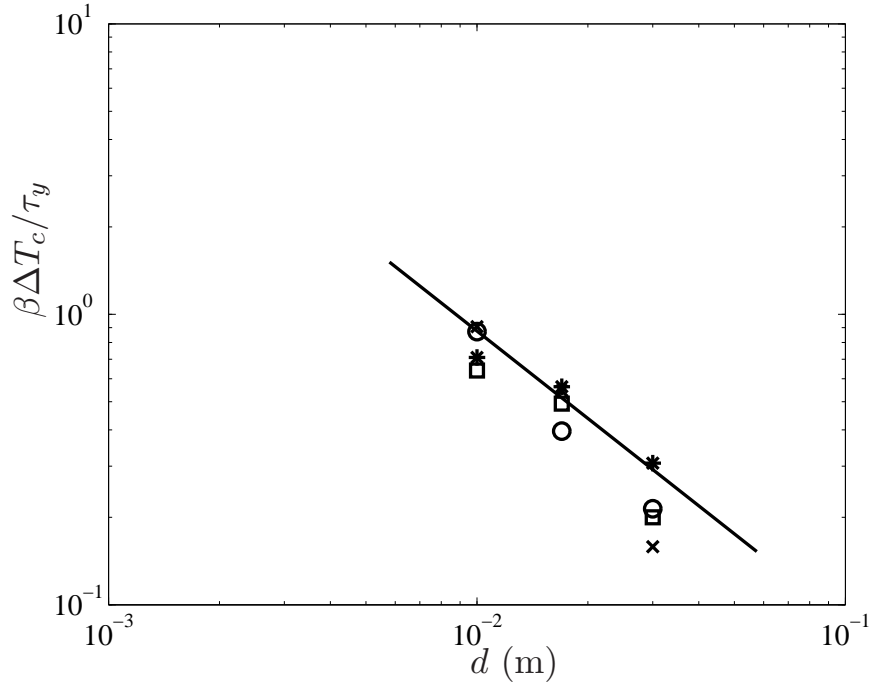

FIG. 9. Variation of $\beta \Delta T_{c} / \tau_{y}$ as a function of $d$ for Carbopol gels $\left(\square: \tau_{y}=0.02 \mathrm{~Pa}, \bigcirc: \tau_{y}=0.008 \mathrm{~Pa}, \times: \tau_{y}=0.002 \mathrm{~Pa}\right.$, *: results obtained by Darbouli et al. $\left.{ }^{24}\right)$ - continuous line: slop -1 .

measurements, when the flow occurs, the stress scale is such as $G^{\prime} \gamma_{c}=\tau_{y}$. Based on SAOS measurements, a viscosity is introduced $\mu^{*}=\frac{\sqrt{G^{\prime 2}+G^{\prime \prime 2}}}{\omega}$ and a characteristic scale for the viscosity can be derived $\mu=\frac{\tau_{y} d^{2}}{\kappa}$, where the diffusion time $d^{2} / \kappa$ is the time scale. By replacing this scale in the Rayleigh number given by Eq. (1), one recovers the inverse of the yield number given by Eq. (2). In this particular case, the inverse of the yield number, i.e. ratio between the buoyancy to the yield stress effects, is a generalized Rayleigh number $R a_{g}$.

(ii) Another possible scenario consists in considering the Rayleigh number for a viscous fluid $R a$ defined in Eq. (1) and in determining a viscosity scale $\mu$ obtained by creep rheometry close to the yield stress. By performing creep measurements, we have obtained a viscosity $\mu^{+}$ which depends on the creep time such as $\mu^{+}=B t^{m}$ with $m \approx 1$. If one considers the diffusion time $d^{2} / \kappa$ as the time scale and $m=1$, then one obtains:

$$
R a=\frac{\rho g \beta \Delta T d^{3-2 m}}{B \kappa^{m-1}}=\frac{\rho g \beta \Delta T d}{B} .
$$

If $m=1$, the dimension of $B$ is the same as a stress. In creep measurements below the yield stress value, $\tau_{y}$ appears as the upper bound for $B$. In this sense, one considers the yield stress as the scale for $B$, i.e. $B=\tau_{y}$ in Eq. (8) leading to the generalized Rayleigh number $R a_{g}=1 / Y$ as defined in Eq. (2).

(iii) In this scenario, we consider the microstructure of the Carbopol gel as a matrix solid (percolated microgels) and a Newtonian fluid (water) all around. For this rea- 
son, we are interested in the Rayleigh-Bénard convection of Newtonian fluids in a porous medium. This configuration has been first investigated by Horton \& Rogers ${ }^{49}$ and Lapwood ${ }^{50}$. In their works, the authors consider a layer of a porous medium confined by two horizontal walls where an adverse temperature gradient is imposed. It is assumed that the medium is homogeneous and isotropic, that the solid matrix is non deformable and that the Darcy's law is valid. By considering these assumptions, the Horton-Rogers-Lapwood (HRL) problem leads to define the Rayleigh-Darcy number $R a_{p}$ which is the product of the Darcy number $k / d^{2}$ and the Rayleigh number (Eq. (1)):

$$
R a_{p}=\frac{k \rho_{l} g \beta_{l} \Delta T d}{\kappa_{l} \mu_{l}}
$$

where the index $l$ stands for the 'liquid' phase. The linear stability analysis of this problem shows that the critical Rayleigh value obtained for solid porous media is 27.10 independently of the permeability $k$ of the medium. This critical value is obtained when considering similar boundary conditions as that of our experiments, i.e. impermeable walls, isothermal and no-slip conditions on the upper wall ; an imposed heat flux and no-slip conditions on the lower wall but considering an infinite layer of porous medium ${ }^{51,52}$. It has been shown by Wang ${ }^{55}$ that the critical Rayleigh-Darcy value can be modified depending on the aspect ratio of the cavity. Nevertheless, Wang ${ }^{55}$ shows that in the range of $\Gamma=2$ to $\Gamma=6$, the critical Rayleigh-Darcy value varies slightly (maximal value of 28.6) compared with the infinite layer case.

In the case of microgels suspensions, considered here as teneous porous media, one has evaluated $R a_{p}$ at criticality with the values of $k$ obtained previously (section IIIB) and by considering water as the liquid phase all around the microgels. Results are displayed in Fig. 10 and they highlight a large difference with the HRL problem since $R a_{p}=O\left(10^{-4}\right)$ in the case of our gels at criticality. Furthermore, results in our gels show that the critical $R a_{p}$ values depend on $k$, hence $\tau_{y}$ as one can notice in Fig. 10. One would have expected an independence of $R a_{p}$ with $k$ (and $\tau_{y}$ ) because the microstructure of the gels remains quite similar in the range of the tested Carbopol concentrations (dispersion of fully swollen microgels). The dependency of $R a_{p}$ with $k$ could imply that the Horton-Rogers-Lapwood problem is not appropriate to the Carbopol case. It leads to the conclusion that the analogy to porous media, at least the analogy to the HRL problem, fails. On the one hand, one could simply think that the analogy fails because the onset of convection is not only characterized by the motion of the solvent but by the motion of the bulk material. It means that the convection would be characterized by the movement of the individual gel particles as well as the motion of water while only the fluid moves in the HRL problem. One could imagine another yield stress fluid, such as weakly aggregated suspensions or hard sphere suspensions above the yield stress concentration occurence (about 50\%) and below the random compact packing concentration (about $64 \%$ ), in which the convection first occurs in the porous structure without any movement of the structural elements. This case would be in a better agreement with the HRL problem.

On the other hand, the comparison between the Rayleigh-Bénard convection in a porous medium made up of percolated microgels and the HRL problem might be audacious. Indeed, one knows that the microgels suspension are elastically deformable contrarily to the porous medium considered in the HRL problem. It means that our system should rather be compared with the Rayleigh-Bénard convection in deformable porous media. However, to our knowledge, the (heat) flow of Newtonian fluids in deformable porous media has been few studied $^{56-61}$ while the Rayleigh-Bénard problem in deformable porous media has never been addressed. In addition, focusing on the permeability measurement, a main assumption used in the HRL problem is that the Darcy law is valid. In the case of microgels suspensions, we could think that the measured permeability is not only the Darcy permeability given by Eq. (4). Since the microgels and the solution are ionized as highlighted by Borrega et al. ${ }^{37}$, further local effects, such as osmotic (and maybe electro-osmotic ${ }^{65}$ ) effects, can have a consequence on the flow conditions as higlighted by several studies $^{62-64}$ and thus on the permeability. The variation of the ionic concentrations during the flow can modify locally the osmotic pressure and can have a consequence on the mean velocity of the solvent flowing through the porous medium ${ }^{63,64}$. These different effects, i.e. the Darcy, the osmotic (and the electro-osmotic) effects are additive when evaluating a mean velocity because each effect introduces an additional permeability. Finally, these complex phenomena could modify the onset of convection in our gels, when considering them as porous media, compared with the case of the RBC of Newtonian fluid in a non deformable solid porous medium such as the one considered in the HRL problem. It means that the Rayleigh-Darcy number defined in Eq. (9) would not be appropriate to the case of a suspension of microgels. Further investigations are required in order to determine the importance of the osmotic (and electro-osmotic) effects in the permeability measure in Carbopol gels as well as to consider the Rayleigh-Bénard problem in a weakly deformable porous medium including local osmotic and electro-osmotic effects.

As a conclusion, one can observe that the both approaches (i) and (ii) lead to recover the governing parameter of the RBC in Carbopol gels which is the (inverse) of the Yield number $Y$. The first approach has already been proposed in the case of slip conditions at walls by Darbouli et $a .^{24}$, it has been confirmed in the light of our new results, obtained in the case of no-slip conditions at walls. The second approach needed further investigations. By measuring the rheological properties of Carbopol gels, we have characterized the instantaneous viscosity $\mu^{+} \sim B t^{1}$. By replacing this viscosity in Eq. 


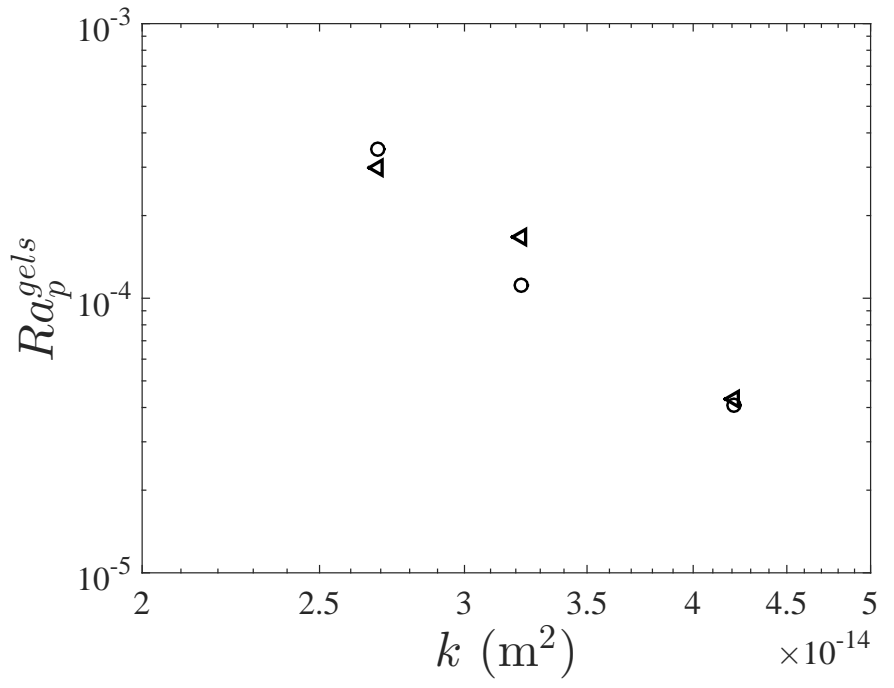

FIG. 10. Critical porous Rayleigh number in the case of Carbopol gels $R a_{p}^{\text {gels }}$ as a function of the permeability $k(\bigcirc$ : $d=17 \mathrm{~mm}, \triangleleft: d=10 \mathrm{~mm}$.)

(1), we have shown that the Yield number can be recovered as the governing parameter. It means that this approach can explain the origin of the onset of $\mathrm{RBC}$ in gels. In other words, this present study confirms that the solid-like to liquid-like transition has to be taken into account in order to understand the onset of motion (convection) in the Carbopol gels. It is of utmost importance to consider more realistic models as the inelastic Bingham or Herschel-Bulkley models up to determine theoretical critical conditions. One thinks to an elastoviscoplastic model as the one proposed by Saramito ${ }^{66,67}$ for instance, or a more complex model which also introduces the viscosity $\mu^{+}$based on the kinematic hardening as proposed by Dimitriou et al. ${ }^{14,68}$.

Finally, by considering Carbopol microgels as a porous medium, approach (iii), for the first time, we have provided permeability values $k=O\left(10^{-14}\right) \mathrm{m}^{2}$, associated to a bulk pressure gradient (Darcy's law), in Carbopol gels. We have also determined a critical value of the Rayleigh-Darcy number in our gels $R a_{p}=O\left(10^{-4}\right)$. One gets that the critical Rayleigh-Darcy number depends on $k$ which is surprising since the range of our gels concentrations leads to a similar microstructure. The comparison with the HRL problem fails in the Carbopol gels because the onset of convection could be characterized by the motion of the bulk material (not only in the fluid first) or also because the Carbopol gels represent a specific porous medium whose properties are far from that of the porous medium considered in the HRL problem. To consider a deformable porous medium including osmotic effects (maybe also electro-osmotic effects) should represent a more relevant comparison and a possible perspective to our work.

\section{ACKNOWLEDGMENTS}

The work of the authors has been supported by the French National Agency of Research (ANR), grant called "ThiM" (ANR-10-BLAN-0925-01). The Laboratoire de Rhéologie et Procédés (LRP) is part of the LabEx Tec21 (Investissements d'Avenir - Grant ANR-11-LABX0030) and the PolyNat Carnot Institut (Investissements d'Avenir - Grant ANR-11-CARN-030-01).

${ }^{1}$ A. Fernandez-Nieves, H. Wyss, J. Mattsson \& D.A. Weitz Microgel suspensions: Fundamentals and Applications Wiley VCH (2011).

${ }^{2}$ Ketz RJ., Prud'homme RK. \& Graessley WW. Rheology of concentrated microgel solutions, Rheol. Acta , 531-539 (1988)

${ }^{3}$ Roberts GP. \& Barnes HA. New measurements of the flow curves for carbopol dispersions without slip artefacts, Rheol. Acta 40, 499-53 (2001)

${ }^{4}$ Piau J.-M Carbopol gels: Elastoviscoplastic and slippery glasses made of individual swollen sponges. Meso- and macroscopic properties, constitutive equations and scaling laws, J.Non-Newtonian Fluid Mech. 144, 1 (2007).

${ }^{5}$ Coussot P., Tabuteau H., Chateau X., Tocquer L. \& Ovarlez G. Aging and solid or liquid behavior in pastes, J. Rheol. 50 (6), 975-994 (2006)

${ }^{6}$ Caton F. \& Baravian C. Plastic behavior of some yield stress fluids: from creep to long-time yield, Rheol. Acta 47, 601-607 (2008)

${ }^{7}$ Oppong F.K., Rubatat L., Frisken BJ., Bailey AE. \& de Bruyn J.R. Microrheology and structure of a yield-stress polymer gel, Phys. Rev. E 73041405 (2006)

${ }^{8}$ Ragouilliaux A., Ovarlez G., Shahidzadeh-Bonn N., Herzhaft B., Palermo T. \& Coussot P. Transition from a simple yield stress fluid to a thixotropic material Phys. Rev. E 76051408 (2007)

${ }^{9}$ Moller P., Fall A., Chikkadi V., Derks D. \& Bonn D. An attempt to categorize yield stress fluid behaviour, Philos. Trans. R. Soc. Math. Phys. Eng. Sci. , 367, 1909, 5139-5155 (2009)

${ }^{10}$ Moller P., Fall A. \& Bonn D. Origin of apparent viscosity in yield stress fluids below yielding, Europhys. Lett., 87, 38004 (2009)

${ }^{11}$ Divoux T., Tamarii D. Barentin C. \& Manneville S. Transient shear banding in a simple yield stress fluid, Phys. Rev. L 104, 208301 (2010)

${ }^{12}$ Divoux T., Barentin C. \& Manneville S. From stress-induced fluidization processes to Herschel-Bulkley behaviour in simple yield stress fluids, Soft Matter 7, 8409-8418 (2011)

${ }^{13}$ Lee D., Gutowski IA., Bailey AE., Rubatat L., de Bruyn J.R. \& Frisken BJ. Investigating the microstructure of a yield stress fluid by light scattering, Phys. Rev. E 83031401 (2011)

${ }^{14}$ Dimitriou C., Ewoldt R.H. \& McKinley G. H. Describing and Prescribing the Constitutive Response of Yield Stress Fluids Using Large Amplitude Oscillatory Shear Stress (LAOStress), J. Rheol. 57 (27), 1-44 (2013)

${ }^{15}$ Lidon P., Villa L. \& Manneville S. Power-law creep and residual stresses in a carbopol gel Rheol. Acta, 56 (3), 307-323 (2017)

${ }^{16}$ Balmforth N., Frigaard I.A. \& Ovarlez G. Yielding to stress: Recent developments in viscoplastic fluid mechanics, Annu. Rev. Fluid Mech. 46, 121-146 (2014)

${ }^{17}$ Bonn D., Parades J., Denn M., Berthier L., Divoux T. \& Manneville S. Yield stress materials in soft condensed matter, arXiv:1502.05281v1. (2015)

${ }^{18} \mathrm{~S}$. Chandrasekhar Hydrodynamic and hydromagnetic stability Clarendon press, Oxford (1961).

${ }^{19}$ E.L. Koschmieder Bénard cells and Taylor vortices Cambridge University press (1993).

${ }^{20}$ Bodenschatz E., Pesch W. \& Ahlers G. Annu. Rev. Fluid Mech. (2000).

${ }^{21}$ Zhang J. , Vola D. \& Frigaard I. A. Yield stress effects on Rayleigh Bénard convection, J. Fluid Mech. 566, 389 (2006). 
${ }^{22}$ Vikhansky A. Thermal convection of a viscoplastic liquid with high Rayleigh and Bingham numbers, Phys. Fluids. 21103103 (2009).

${ }^{23}$ Balmforth N.J. \& Rust A. Weakly nonlinear viscoplastic convection, J. Non-Newtonian Fluid Mech. 158, 36 (2008).

${ }^{24}$ Darbouli M, Metivier C, Piau JM, Magnin A \& Adbelali A. Rayleigh-Bénard convection for viscoplastic fluids, Phys. Fluids. 25:023101 (2013).

${ }^{25}$ Kebiche Z., Castelain C. \& Burghelea T. Experimental investigation of the Rayleigh-Bénard convection in a yield stress fluid, J. Non-Newtonian Fluid Mech. 203, 9-23 (2014)

${ }^{26}$ Cloitre M \& Bonnecaze R.T. A review on wall slip in high solid dispersions, Rheol. Acta (56) 283-305 (2017).

${ }^{27}$ Davaille A., Gueslin B, Massmayer A. \& Di Giuseppe E. Thermal instabilities in a yield stress fluid: Existence and morphology, J. Non-Newtonian Fluid Mech. 193, 144-153 (2013)

${ }^{28}$ Karimfazli I. , Frigaard I.A. \& Wachs A. Thermal plumes in viscoplastic fluids: flow onset and development, J. Fluid Mech. 787, 474-507 (2015).

${ }^{29}$ Massmayer A., Di Giuseppe E., Davaille A., Tobias R. \& Tackley P.J. Numerical simulation of thermal plumes in a HerschelBulkley fluid, J. Non-Newtonian Fluid Mech. 195, 32-45 (2013)

${ }^{30} \mathrm{Li}$ C., Magnin A. \& Métivier C. Natural convection in shearthinning yield stress fluids in a square enclosure, Aiche J. 62 (4), 1347-1355 (2016)

${ }^{31}$ de Souza Mendes P.R. \& Dutra E.S.S. Viscosity function for yield stress liquids, Appl. Rheol. 14, 296-302 (2004)

${ }^{32}$ Frigaard I.A. \& Nouar C. On the usage of viscosity regularisation methods for viscoplastic flow computation J. Non-Newtonian Fluid Mech. 127 (1), 1-26 (2005)

${ }^{33}$ Métivier C. \& Nouar C. Stability of a Rayleigh-Bénard Poiseuille flow for yield stress fluids - Comparison between Bingham and regularized models Int. J. Non-Linear Mech. 46, 1205-1212 (2011)

${ }^{34}$ Cerisier P., Rahal S., Cordonnier J. \& Lebon G. Thermal influence of boundaries on the onset of Rayleigh-Bénard convection, Int. J. Heat Transfer 41, 3309-3320 (1998)

${ }^{35}$ Gershuni G.Z., Zhukhovitskii E.M \& Semakin I.G. On convective instability in a horizontal fluid layer separating walls of different conductivity, Uchen Zap perm Univ 248, 18-29 (1967)

${ }^{36}$ Gershuni G.Z. \& Zhukhovitskii E.M. Convective stability of incompressible fluids. Jerusalem: Israel Programm for Scientific Translations p. 32 (1976)

${ }^{37}$ Borrega R., Cloître M., Betremieux I., Ernst B. \& Leibler L. Concentration dependence of the low-shear viscosity of polyelectrolyte micro-networks: form hard spheres to soft microgels, Europhys. Lett., 47 (6), 729-735 (1999)

${ }^{38}$ Mougin N., Magnin A. \& J.-M. Piau The significant influence of internal stresses on the dynamics of bubbles in a yield stress fluid, J.Non-Newtonian Fluid Mech. 171-172, 42-55 (2012).

${ }^{39}$ Andrade E.N. da C. On the viscous flow in metals and allied phenomena, Proc. R. Soc. A 84 (1), 1-12 (1910)

${ }^{40}$ Nechad H., Helmstetter A., El Guerjouma R. \& Sornette D. Creep ruptures in heterogeneous materials, Phys. Rev. L 94, 045501 (2005)

${ }^{41}$ Miguel M.-C., Vespignai A., Zaiser M. \& Zapperti S. Dislocation jamming and Andrade creep, Phys. Rev. L 89 (16) (2002)

${ }^{42}$ Liu A.J. \& Nagel S.R. Jamming is not just cool any more, Nature (London) 396, 21 (1998)

${ }^{43}$ Ambari A., Amiel C., Gauthier-Manuel B. \& Guyon E. An experimental measurement of the permeability of deformable porous media, Revue Phys. Appl. 21, 53-58 (1986)

${ }^{44}$ Lelievre V. Rhéologie et filtration de dispersions aqueuses de nanoparticules d'hectorite en relation avec la structuration des dépôts, PhD, Grenoble, INPG (2005)
${ }^{45}$ Weiss N. \& Silberberg A. Hydrogels for Medical and Related Applications, Chap.5, ACS Symposium Series No. 31, Washington (1976).

${ }^{46}$ Weiss N. \& Silberberg A. Inhomogeneity of Polyacrylamide gel structure from permeability and viscoelasticity, Br. Polym. J. 9, 144-150 (1977)

${ }^{47}$ Grattoni C.A., Al-Sharji H.H., Yang C., Muggeridge A.H. \& Zimmerman R.W. Rheology and permeability of crosslinked polyacrylamide gel, J. Colloid Int. Sci. 240, 601-607 (2001)

${ }^{48} \mathrm{Gu}$ W.Y., Yao H., Huang C.Y. \& Cheung H.S. New insight into deformation-dependent hydraulic permeability of gels and cartilage, and dynamic behavior of agarose gels in confined compression, J. Biomech. 36, 593-298 (2003)

${ }^{49}$ Horton C.W. \& Rogers F.T. Convection currents in a porous medium, J. App. Phys. 16, 367-370 (1945)

${ }^{50}$ Lapwood E.R. Convection of a fluid in a porous medium, Proc. Camb. Phil. Soc. 44, 508-521 (1948)

${ }^{51}$ Nield D.A. Onset of thermohaline convection in a porous medium, Water Resources Res. 11, 553-560 (1968)

${ }^{52}$ Nield D.A. \& Bejan A. Convection in porous media, SpringerVerlag, New-York (2006)

${ }^{53}$ Métivier C., Rharbi Y., Magnin A. \& Bou Abboud A. Stick-slip control of the Carbopol gels on transparent smooth walls, Soft Matter, 8, 7365 (2012).

${ }^{54}$ Schmidt R.J. \& Milverton S.W. On the instability of a fluid when heated from below, Proc. Roy. Soc. (London)A. 152, (1935).

${ }^{55}$ Wang C.Y. Onset of natural convection in a fluid-saturated porous medium inside a cylindrical enclosure bottom heated by constant flux, Int. Comm. Heat Mass Transfer 25, 593-598 (1998)

${ }^{56}$ Biot M. General theory of three-dimensional consolidation, J. Appl. Phys. 12, 155-164 (1941)

${ }^{57}$ Ghaboussi J. \& Wilson E.L. Flow of compressible fluid in porous elastic media, Int. J. Numer. Meth. Engng 5, 419-442 (1973)

${ }^{58}$ Lewis R.W. \& Schrefler B.A. The Finite element method in the deformation and consolidation of porous media, Wiley, New York (1987)

${ }^{59}$ Detournay E. \& Cheng A. H-D. Poroelastic response of a borehole in a non-hydrostatic stress field, Int. J. Rock Mech. Min. Sci. and Geomech. Abstr. 25 (3), 171-182 (1988)

${ }^{60}$ Spiegelman M. Flow in deformable porous media. Part1 Simple analysis, J. Fluid Mech. 247, 17-38 (1993)

${ }^{61}$ Bai M. \& Roegiers J.C. Fluid flow and heat flow in deformable fractured porous media, Int. J. Engng Sci. 32 (10), 1615-1633 (1994)

${ }^{62} \mathrm{Gu}$ W., Lai W. \& Mow V. A mixture theory for charged-hydrated soft tissues containing multi-electrolytes: Passive transport and swelling behaviors, J. Biomech. Engrg. 120, 169-180 (1998)

${ }^{63}$ Moyne C. \& Murad M.A. Electro-chemo-mechanical couplings in swelling clays derived from a micro/macro-homogenization procedure, Int. J. Solids Struc. 39, 6159-6190 (2002)

${ }^{64}$ Lemaire T. Couplages électro-chimio-hydro-mécaniques dans les milieux argileux. PhD thesis,INPL, Nancy, France (2004)

${ }^{65}$ Boscus J. Transfert électro-osmotique en milieu poreux déformable - application au gel d'agar-agar, $\mathrm{PhD}$, Université de Montpellier (2005)

${ }^{66}$ Saramito P. A new constitutive equation for elastoviscoplastic fluid flows, J. Non-Newtonian Fluid Mech. 145 (1), 1-14 (2007)

${ }^{67}$ Saramito P. A new elastoviscoplastic model based on the Herschel-Bulkley viscoplastic model, J. Non-Newtonian Fluid Mech. 158, 154-161 (2009)

${ }^{68}$ Dimitriou C. \& McKinley G. H. A comprehensive constitutive law for waxy crude oil: a thixotropic yield stress fluid, Soft Matter 10, 6619-6644 (2014) 